\title{
Watching Movies Unfold, a Frame-by-Frame Analysis of the Associated Neural Dynamics
}

\author{
Anna M. Monk, Daniel N. Barry, Vladimir Litvak, @Gareth R. Barnes, and $\mathbb{D}^{\mathbb{E}}$ Eleanor A. Maguire
}

https://doi.org/10.1523/ENEURO.0099-21.2021

Wellcome Centre for Human Neuroimaging, University College London Queen Square Institute of Neurology, University College London, London WC1N 3AR, United Kingdom

\begin{abstract}
Our lives unfold as sequences of events. We experience these events as seamless, although they are composed of individual images captured in between the interruptions imposed by eye blinks and saccades. Events typically involve visual imagery from the real world (scenes), and the hippocampus is frequently engaged in this context. It is unclear, however, whether the hippocampus would be similarly responsive to unfolding events that involve abstract imagery. Addressing this issue could provide insights into the nature of its contribution to event processing, with relevance for theories of hippocampal function. Consequently, during magnetoencephalography (MEG), we had female and male humans watch highly matched unfolding movie events composed of either scene image frames that reflected the real world, or frames depicting abstract patterns. We examined the evoked neuronal responses to each image frame along the time course of the movie events. Only one difference between the two conditions was evident, and that was during the viewing of the first image frame of events, detectable across frontotemporal sensors. Further probing of this difference using source reconstruction revealed greater engagement of a set of brain regions across parietal, frontal, premotor, and cerebellar cortices, with the largest change in broadband $(1-30 \mathrm{~Hz})$ power in the hippocampus during scene-based movie events. Hippocampal engagement during the first image frame of scene-based events could reflect its role in registering a recognizable context perhaps based on templates or schemas. The hippocampus, therefore, may help to set the scene for events very early on.
\end{abstract}

Key words: ERFs; hippocampus; MEG; movie events; scenes; sequences

\section{Significance Statement}

Our experience of the world is much like watching a movie. Although it appears to be seamless, it is in fact composed of individual image frames that we perceive between eye blinks. The hippocampus is known to support event processing, but questions remain about whether it is preferentially involved only when events are composed of scenes that reflect the real world. We found that a set of brain regions including the hippocampus was engaged during the first image frame of scene-based events compared with highly matched events composed of abstract patterns. This suggests that the hippocampus may set the scene for an event very early on.

\section{Introduction}

We generally perceive the world as a series of visual snapshots punctuated by eye blinks and saccades. With parallels in terms of how the individual frames of a movie appear to be continuous (Tan, 2018), somehow these separate

Received March 14, 2021; accepted June 2, 2021; First published June 28, 2021.

The authors declare no competing financial interests. images become linked together, such that we have a sense of the seamless unfolding of life and events (Cutting, 2005; Magliano and Zacks, 2011). These dynamic events are central to our lived experience, be that during "online" perception, or when we recall the past or imagine the future.

Author contributions: A.M.M. and E.A.M. designed research; A.M.M. performed research; A.M.M., D.N.B., V.L., G.R.B., and E.A.M. analyzed data; A.M.M. and E.A.M. wrote the paper. 
Here, we defined an event as a dynamic, unfolding sequence of actions that could be described in a story-like narrative. Functional MRI (fMRI) has helped delineate the brain areas involved in supporting event processing (Zacks et al., 2001; Hasson et al., 2008; Lehn et al., 2009; Summerfield et al., 2010; Reagh et al., 2020), a salient example being the events captured in autobiographical memories. When people recollect these past experiences, a distributed set of brain areas is engaged, including the hippocampus, parahippocampal, retrosplenial, parietal, and ventromedial prefrontal cortices (Maguire, 2001; Svoboda et al., 2006; Cabeza and St Jacques, 2007; Spreng et al., 2009). Interestingly, two key elements of events, individual scene snapshots (Hassabis et al., 2007a; Zeidman et al., 2015) and sequences (Kumaran and Maguire, 2006; Lehn et al., 2009; Schapiro et al., 2016; Liu et al., 2019), engage several of the same brain regions, including the hippocampus. Aligning with these fMRI findings, impairments in recalling past events (Scoville and Milner, 1957; Rosenbaum et al., 2005; Kurczek et al., 2015), imagining single scene images (Hassabis et al., 2007b), and processing sequences (Mayes et al., 2001; Dede et al., 2016) have been documented in patients with bilateral hippocampal damage.

While much has been learned about event processing from fMRI and neuropsychological studies, we still lack knowledge about the precise temporal dynamics associated with unfolding events. This is not surprising given the temporal lag of the fMRI BOLD signal. By contrast, the finer temporal resolution of evoked responses measured by magnetoencephalography (MEG) and electroencephalography (EEG) offers a means to establish the millisecond-by-millisecond neural dynamics associated with events as they evolve. There are relatively few MEG/EEG studies of event processing. Investigations have typically used viewing of movies or television shows as event stimuli to examine the consistency of neural activity patterns across participants (Lankinen et al., 2014; Chang et al., 2015; Betti et al., 2018; Chen and Farivar, 2020), or to assess segmentation of such stimuli into discrete events (Silva et al., 2019). However, the fundamental underlying temporal dynamics of event processing remain essentially unaddressed.

Extended events, as represented in movies or autobiographical memories, involve visual imagery from the real world and, as noted, the hippocampus is frequently engaged in this context. It is unclear, however, whether it would be similarly responsive to unfolding events that involve abstract imagery. One theoretical position suggests

This work was supported by the Wellcome Principal Research Fellowship 210567/Z/18/Z (to E.A.M.) and the Centre by the Wellcome Award 203147/Z/ $16 / Z$.

Acknowledgements: We thank Daniel Bates, David Bradbury, and Eric Featherstone for technical support and Zita Patai for analysis advice.

Correspondence should be addressed to Eleanor A. Maguire at e.maguire@ ucl.ac.uk.

https://doi.org/10.1523/ENEURO.0099-21.2021

Copyright @ 2021 Monk et al.

This is an open-access article distributed under the terms of the Creative Commons Attribution 4.0 International license, which permits unrestricted use, distribution and reproduction in any medium provided that the original work is properly attributed. that the hippocampus may be especially attuned to scenes (Maguire and Mullally, 2013), which we define simply as individual visual images reflecting the real world. Consequently, here, we compared the watching of closely-matched scene-based movie events and nonscene movie events during MEG, with a particular interest in the hippocampal response.

To do this, we created a set of short, simple cartoonlike movies each of which depicted an event. Each event was a self-contained vignette, and was composed of a series of individual images. These individual images were linked such that each image led on to the next, thereby depicting an activity that unfolded over $22.4 \mathrm{~s}$. In essence, the events were digital versions of a flip-book, composed of individual images which, when presented as a sequence, showed the progression of an activity. We devised two types of movie events. In one, each image frame within a movie was a simple scene reflecting the real world, while in the other condition each image frame comprised abstract shapes. The two event types were visually very similar, and both involved unfolding sequences of individual image frames that could be described in a story-like narrative. By leveraging the high temporal resolution of MEG, we could examine each image, allowing us to better understand how a sequence of separate images evolves neurally to give rise to the experience of a seamless event. Moreover, by comparing the two event types, we could address the question of whether or not the hippocampus was preferentially engaged by scenebased events, with relevance for theories of hippocampal function.

\section{Materials and Methods}

\section{Participants}

Twenty-one healthy human participants (11 females; mean age 23.42 years, SD 4.51) took part in the study. All participants had normal or corrected vision, and provided written informed consent to participate in the study (protocol \#1825/005) as approved by the local Research Ethics Committee.

\section{Stimuli}

Short visual movies of events were created by hand using the animation program Stykz 1.0.2 (https://www. stykz.net), each composed of 16 individually drawn image frames presented in a sequence. Each of these 16-image movies lasted $22.4 \mathrm{~s}$. Each event was self-contained and was not part of a larger story. An image comprised a combination of straight lines and circles that created simple line imagery that was easily interpretable. Images were all grayscale to keep the luminance contrast low, and this was invariant across frames and between conditions. A pixelated gray background for all frames was created in the image manipulation software program GIMP 2.8 (https://www.gimp.org).

There were two main stimulus types (Fig. 1A, upper two panels). In one case, each image frame within a movie event was a simple scene (pictures), while in the other, each image frame was composed of abstract shapes 
A
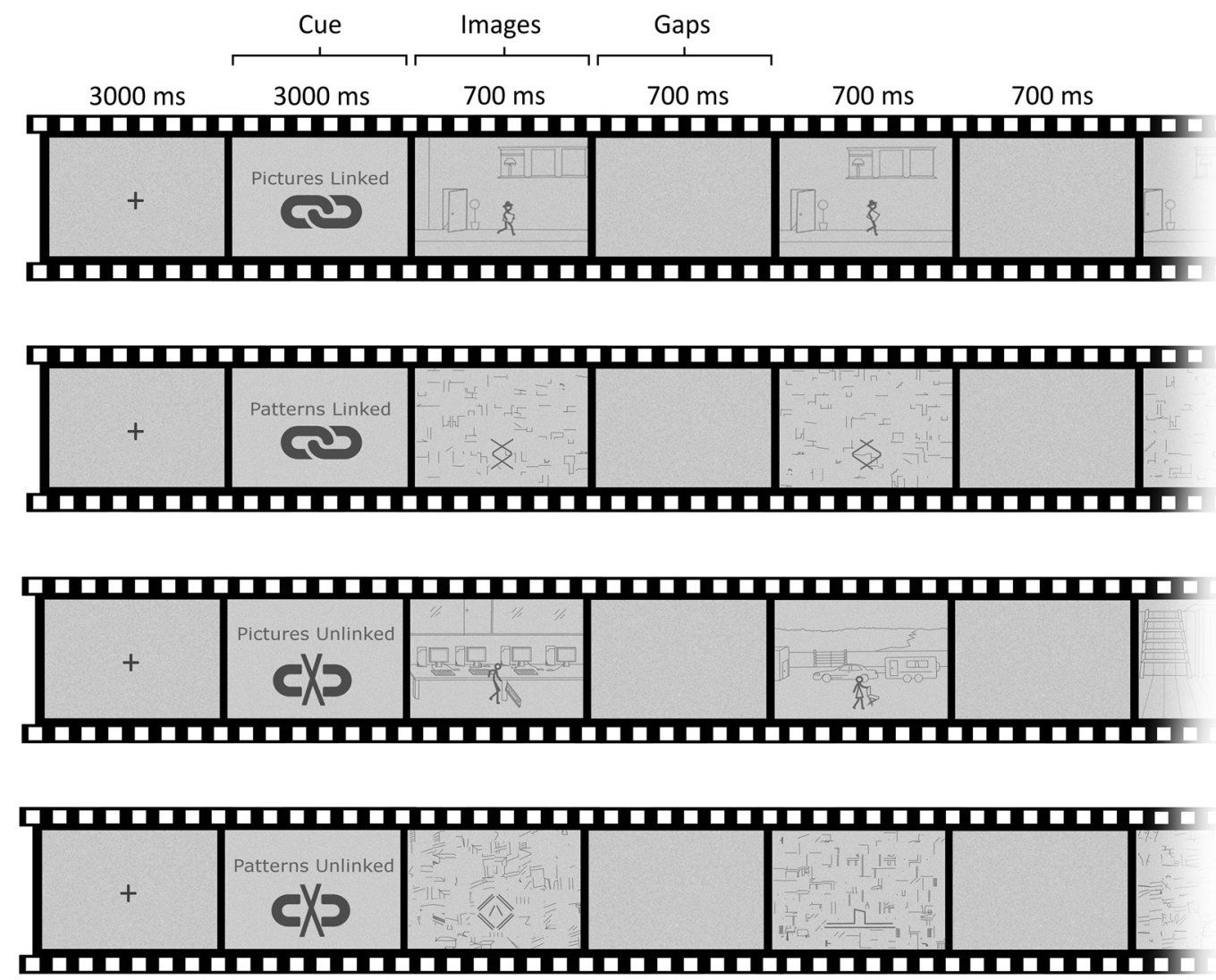

whole clip 22,400 ms

B

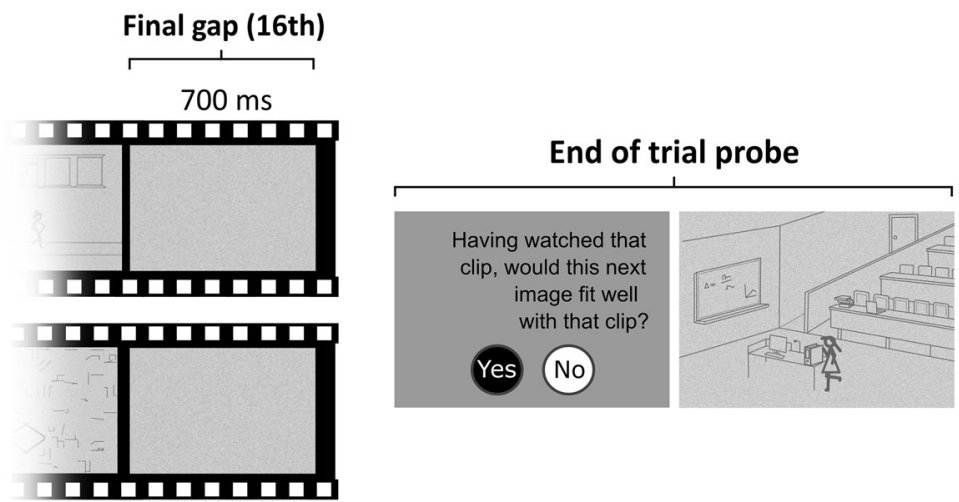

Figure 1. Experimental paradigm. $\boldsymbol{A}$, Schematic of the trial structure common to all movies, with examples of each condition. Each image frame was followed by a gap frame. $\boldsymbol{B}$, A probe question occasionally followed the completion of a clip to assess participants' engagement with the task.

(patterns). In both cases, incremental changes in the stimuli image-to-image showed a progression of activity that connected every image frame over the course of a 22.4-s clip, resulting in two event conditions called pictureslinked and patterns-linked. Pictures-linked movies contained temporally related scenes unfolding over time such that a scene-based event was perceived. A stick-figure character in the center of the image performed a series of brief activities involving a single object that appeared in every frame of the movie. Each movie clip portrayed a different stick-figure paired with a different object with which they interacted. The environment for each movie was a simple representation of an indoor ( $50 \%$ of clips) or outdoor (50\% of clips) scene. Other background objects were included to give a sense of perspective and movement. Patterns-linked movies contained temporally unfolding patterns that matched the evolving nature of pictures-linked movies, where each showed a novel abstract shape that underwent numerous simple mechanical changes, such as a rotation in a particular direction. The 
result was that a non-scene event was perceived. See https://vimeo.com/megmovieclips for dynamic examples of the movies.

The activity depicted in an event could be subdivided into steps. For example, for the event where the overall activity was skate-boarding (see https://vimeo.com/ megmovieclips), the individual steps included: (1) the stick-figure skate-boards over a ramp; (2) lands on the ground; (3) skate-boards on the ground past some shopping carts; and (4) steps off the skate-board and picks it up. Each of these steps, or "subevents," were $\geq 1 \mathrm{~s}$ in duration. Each of the patterns-linked events also contained subevents. For the example provided here: https://vimeo. com/megmovieclips, (1) three diamond shapes are nested within each other; (2) the three diamond shapes start to separate out; (3) the three diamonds become separate and the lines comprising the diamonds become thicker; and (4) the diamond shapes all rotate to the right.

There was one-to-one matching between the stimuli of the two conditions. For each image frame in a patternslinked movie, the number of pixels composing the central shape was matched with the number of pixels composing the stick-figure and its paired object from a corresponding pictures-linked movie. Pictures-linked background images (minus the stick-figure and object) were scrambled to form the individual backgrounds of patterns-linked image frames. The number of frames it took for a particular pattern's movement to unfold (e.g., completion of one rotation of a shape) corresponded to the same number of frames it took for a stick-figure to accomplish a subactivity (e.g., the stick-figure skate-boarded over a ramp), so that the pace of subevents was matched between conditions. An average of four subevents occurred per linked movie. There were 10 unique movies for each stimulus type.

There were two control conditions (Fig. 1A, lower two panels), each with 10 movie clips. Each movie was composed of a series of unique and separate unrelated image frames such that no evolving event could be conceived. Pictures-unlinked movies contained separate scenes for each image frame, and in total there were 160 unique scenes, twenty different stick-figures and 152 unique objects. For example, in one pictures-unlinked movie (see web link), the first image shows a stick-figure in an Internet café, the next image shows a different stick-figure in a trailer park, and each of the remaining images are similarly unrelated to one another. Patterns-unlinked movies were composed of a series of unrelated abstract shapes. In total, there were 160 unique shapes. The same direct frame-to-frame matching procedure used for the linked movies was applied to unlinked movies in terms of the corresponding pixel count of central items and scrambled backgrounds of each image (see https:// vimeo.com/megmovieclips for example stimuli).

In each condition every image frame was presented for $700 \mathrm{~ms}$, a duration identified by piloting as being long enough to comprehend the scene or pattern being viewed, and brief enough to minimize saccades and limit fixations to the center of frames. Between each image, "gap" frames of the same duration were inserted, where no image was displayed and which consisted of only a pixelated gray background (see Fig. 1). The pixelation served to mask the visual persistence of the preceding image. Since images were presented in a sequence, the primary function of gaps was to act as a temporal separator so that individual images could be subjected to analysis independently. Gaps also ensured images in unlinked movies were clearly perceived as independent, and the inclusion of gaps in the linked movies ensured close matching. The 16 gaps matched the number of images in each movie clip, and each movie ended with a gap.

Pilot testing of a larger number of stimuli ensured that we only included in the main experiment those patterns movies that were not interpreted as depicting real objects, scenes, or social events. We also confirmed that the gaps between images did not interrupt the naturalistic comprehension of linked movies or their sense of unfolding. During piloting, each individual movie was also rated on: perceived linking, how linked (or disconnected) images appeared to be; and thinking ahead, how much of the time people found themselves thinking about what might happen next. A significant Friedman test for perceived linking $\left(n=7 ; \chi^{2}{ }_{(3)}=18, p=0.0004\right)$ followed by Wilcoxon signed-rank tests found no significant difference between the two linked conditions $(Z=-0.314, p=0.753)$, or between the two unlinked conditions $(Z=-0.368, p=0.713)$. There was, as expected, a significant effect of linking when comparing pictures-linked with pictures-unlinked $(Z=2.371, p=0.018)$, and patterns-linked with patterns-unlinked $(Z=2.366, p=0.018)$. Similarly, for perceived thinking ahead, a significant Friedman test $\left(\chi^{2}{ }_{(3)}\right.$ $=17.735, p=0.0005$ ) was followed by Wilcoxon signedrank tests that revealed no significant difference between the two linked conditions $(Z=-0.169, p=0.866)$, or between the two unlinked conditions $(Z=-0.271$, $p=0.786)$. There was a significant effect of thinking ahead when comparing pictures-linked with picturesunlinked $(Z=2.371, p=0.018)$, and patterns-linked with patterns-unlinked $(Z=2.366, p=0.018)$.

\section{Prescan training}

Participants were trained before the MEG scan to ensure familiarity with the different conditions and the rate of presentation of movie frames. These practice movies were not used in the main experiment. Specifically, participants were shown examples and told: "The movie clips are made up of a series of images. Some clips have images that are clearly linked to each other, and some have images that are not linked at all, so the images are completely unrelated to one another. The images can be either pictures with a stick-figure character, or abstract patterns." For pictures-linked movies, it was explained "...as you can see, there is a stick-figure character doing something. You'll have noticed that the pictures are all linked together so that the clip tells a story." For patterns-linked movies it was explained: "...for this type of clip, the patterns are all linked together, so one pattern leads to the next one in the clip. In this example clip the pattern moved outwards at first, then the crosses became larger, and then the circles increased in size, then the pattern 
changed again. The shape changed a bit step-by-step so that the clip portrays an evolving pattern." Participants were instructed not to link the images in unlinked movies and to treat each image frame separately when viewing them.

Movies were preceded by one of four visual cues: pictures-linked, patterns-linked, or, for the control conditions, pictures-unlinked and patterns-unlinked (Fig. 1A), to advise a participant of the upcoming condition. Cues were provided in advance of each movie so that participants would not be surprised to discover the nature of the clip. Without a cue, the experiment would be poorly controlled since there would most likely be differences across participants in terms of when they registered the clip type during its viewing. This would make it impossible to timelock processing of the clip to neural activity in a consistent manner across participants. Instead, by using an informative cue, we could be sure that from the very first image frame a participant understood whether the movie was to be composed of linked images or not, and whether these images would depict pictures or patterns.

\section{Task and procedure}

Scripts run in MATLAB R2018a were used to present stimuli and record responses in the MEG scanner. Each trial was preceded by a cue advising of the upcoming condition (e.g., pictures-linked) which was shown for $3000 \mathrm{~ms}$. Each movie was $22,400 \mathrm{~ms}$ in duration from the appearance of the first image frame to the end of the final gap frame (Fig. 1A). Individual image and gap frames were each $700 \mathrm{~ms}$ in duration. Participants then saw a fixation cross for $3000 \mathrm{~ms}$ before the next cue. To ensure participants attended to the movies throughout the scanning session, an occasional probe question was included (two trials per condition; Fig. 1B). Following the final gap frame of a movie, a novel image was presented (either a picture or a pattern) and participants were asked whether this image fitted well with the movie clip they just saw. Of the two probe trials per condition, one was a "yes" trial (the image was congruent with the movie), and one was a "no" trial (the image was incongruent with the movie).

Given the rate at which frames were presented, we sought to minimize a systematic relationship between spontaneous blinking and stimulus onset. Furthermore, fatigue is known to increase blink duration, which could result in participants missing individual frames, and increase the risk of significant head movement. Consequently, to ensure participants remained alert, the scanning session was split into five blocks each lasting $\sim 6 \mathrm{~min}$. During breaks between recordings participants were instructed to blink and rest. Each recording block contained eight movie trials where conditions were presented in a randomized order for each participant. Participants were instructed to maintain fixation in the center of frames during the entire trial and to restrict eye movements to between-trial periods.

In summary, movies were visually similar, with one-toone matching between the two linked and also the two unlinked conditions. Common to all movies was the use of a central item per image, the inclusion of interleaved gap frames, use of simple line illustrations of pictures or patterns in grayscale, all of which were presented at the same frame rate of 1.43 frames per second.

\section{In-scanner eye tracking and analysis}

An Eyelink 1000 Plus (SR Research) eye tracking system with a sampling rate of $2000 \mathrm{~Hz}$ was used during MEG scanning to monitor task compliance and record data ( $x$ and $y$ coordinates of all fixations) across the full screen. The right eye was used for a 9-point grid calibration, recording and analyses. For some participants the calibration was insufficiently accurate, leaving 16 datasets for eye tracking analyses. The Eyelink Data Viewer (SR Research) was used to examine fixation locations and durations. We used the built-in online data parser of the Eyelink software whereby fixation duration was parsed automatically with fixations exceeding $100 \mathrm{~ms}$. Eye tracking comparisons involving all four conditions were performed to examine where (using group eye fixation heat maps) and for how long (using a two-way repeated measures ANOVA) participants fixated during a 700-ms time window. Our primary focus was on comparing the neural activity evoked during the pictures-linked and patterns-linked conditions. Consequently, the outcome of this comparison directed our subsequent examination of the eye tracking data, meaning that we focused the eye tracking analysis on the specific time windows where differences in the neural data were identified. This allowed us to ascertain whether the neural differences between conditions could have been influenced by oculomotor disparities.

\section{Postscan surprise memory test}

Following the experiment, participants completed a surprise free recall test for the event movies, since the principal aim was to examine the neural differences between pictures-linked and patterns-linked movies. Participants were asked to recall everything they could about what happened in each of these clips, unprompted. If they correctly recalled the simple story, they scored "1" for that clip, otherwise they scored " 0 ." Specifically, a score of 1 was awarded if all of the following information was provided: a description of the main figure (be it a stick-figure or abstract pattern) and context, and a narrative containing all of the subevents that unfolded. The maximum score per participant and event condition was therefore 10 (as there were 10 movies per condition). Performance for pictures-linked and patterns-linked were compared using a paired-samples $t$ test with a statistical threshold of $p<0.05$.

\section{MEG data acquisition}

MEG data were acquired using a whole-head 275-channel CTF Omega MEG system within a magnetically shielded room with a sampling rate of $1200 \mathrm{~Hz}$. Participants were scanned in a seated position, with the back of their head resting on the back of the MEG helmet. Head position fiducial coils were attached to the three standard fiducial points (nasion, left and right preauricular) to monitor head position continuously throughout acquisition. 
As noted above, we were particularly interested in hippocampal neural activity. The ability of MEG to detect deep sources, including the hippocampus, has been previously debated (Mikuni et al., 1997; Shigeto et al., 2002). While it is inevitable that spatial resolution decreases with depth (Hillebrand and Barnes, 2002), evidence has accumulated to convincingly establish that MEG can indeed localize activity to the hippocampus (Meyer et al., 2017; Pu et al., 2018; Ruzich et al., 2019). This includes during autobiographical memory event retrieval (McCormick et al., 2020), imagination (Barry et al., 2019; Monk et al., 2021), and memory encoding (Crespo-García et al., 2016). Separate fMRI, MEG, and intracranial EEG (iEEG) studies using the same virtual reality paradigm have also revealed similar hippocampal (theta) activity across modalities (Doeller et al., 2008; Kaplan et al., 2012; Bush et al., 2017). Particularly compelling are studies using concurrent MEG and iEEG, where the ground truth is available, that have demonstrated MEG can successfully detect hippocampal activity using beamforming (CrespoGarcía et al., 2016). We were, therefore, confident that we could record neural activity from the hippocampus.

\section{MEG data preprocessing}

MEG data were preprocessed using SPM12 (www.fil. ion.ucl.ac.uk/spm). Data were high-pass filtered at $1 \mathrm{~Hz}$ to eliminate slow drifts in signals from the MEG sensors. A band-stop filter was applied at $48-52 \mathrm{~Hz}$ to remove the power line interference, and at $98-102 \mathrm{~Hz}$ to remove its first harmonic. Epochs corresponding to each movie cue were defined as $-100-1000 \mathrm{~ms}$ relative to cue onset. Image frames were defined as $-100-700 \mathrm{~ms}$ relative to image onset. Gap periods were defined as $-100-700 \mathrm{~ms}$ relative to gap onset. Epochs were concatenated across trials for each condition, and across scanning sessions. Before the calculation of event-related fields (ERFs), data were first low-pass filtered using a two-pass sixth order Butterworth filter, with a frequency cutoff of $30 \mathrm{~Hz}$. We implemented a broadband approach $(1-30 \mathrm{~Hz})$, since the focus of this experiment was evoked activity. Although activity within the theta band $(4-8 \mathrm{~Hz})$ is often associated with the hippocampus (Colgin, 2013, 2016), there is also evidence for the role of alpha $(9-12 \mathrm{~Hz})$ and beta (13-30 $\mathrm{Hz}$ ) power in event processing (Hanslmayr and Staudigl, 2014). Following visual inspection of the data, an average of only 0.76 epochs were discarded on the basis of containing eye blinks and muscle artifacts. To baseline-correct, the activity during the first $1000 \mathrm{~ms}$ from the onset of the fixation period was averaged and subtracted from each cue, image or gap epoch. The robust average was calculated to obtain an ERF per participant and condition. This averaging method down-weights outliers when computing the average and helps to suppress high-frequency artifacts and minimizes trial rejection (Wager et al., 2005).

\section{MEG data analyses}

Our principal aim was to assess differences between the pictures-linked and patterns-linked conditions since our main interest was in comparing the processing of events built from scenes with those built from nonscenes. In order to make this key comparison, our focus was on the individual image frames that composed the movies. As previously mentioned, gaps were included in the design to provide temporal separation between images, so that brain activity associated with each movie image could be separately examined without interference or leakage from the previous image. Consequently, we explored both the evoked responses to particular images along the time course of the movies and then, as a second step, the likely sources of these responses. These steps are described below.

\section{ERF analysis}

ERFs were analyzed using the FieldTrip toolbox (Oostenveld et al., 2011), implemented in MATLAB $\mathrm{R} 2018 \mathrm{a}$, on the robust averaged data per condition. A targeted sliding window approach was used to examine differences between the two event conditions within salient time windows during movies, namely images 1,2 , 8, and 16. At image 1 , only this first single image of a sequence was being viewed; at image 2 , there was already the context set by the preceding first image; image 8 represented the mid-point of a sequence; and image 16 was the final image. This approach enabled sampling across a long clip length, while also minimizing multiple comparisons. A number of secondary contrasts involving the premovie cues, the gap frames, and control conditions were also performed to examine whether any differences observed between the two event conditions could be explained by other factors.

We used a non-parametric cluster-based permutation approach for our ERF analyses, a commonly adopted approach that deals with the multidimensional nature of MEG (and EEG) data (see Maris, 2004, 2012; Maris and Oostenveld, 2007). Cluster-based correction addresses both issues of correlation (since electrophysiological responses are necessarily correlated) and multiple comparisons, balanced with maximizing the sensitivity to detect an effect in multidimensional data. The cluster-based permutation approach corrects for multiple comparisons across all MEG channels and time samples across a specific time window. It also controls for the Type I error rate by identifying clusters of significant differences over time and sensors rather than performing separate tests for each sample of time and space. This makes it a particularly powerful approach for MEG/EEG data, and a statistically robust method to determine time windows and sensor locations of effects.

Specifically, each pairwise comparison was performed using the non-parametric cluster-based permutation test, providing a statistical quantification of the sensor-level data while correcting for multiple comparisons across all MEG channels and time samples (Maris and Oostenveld, 2007), across the first $1000 \mathrm{~ms}$ of the cue and the entire $700 \mathrm{~ms}$ of images and gaps. Cluster-level statistics are the sum of $t$ values within each cluster, and this was calculated by taking the maximum cluster-level statistic (positive and negative separately), over 5000 random permutations of the observed data. The obtained $p$ value 
represents the probability under the null hypothesis (no difference between a pair of conditions) of observing a maximum greater or smaller than the observed clusterlevel statistics. We report only effects that survived this correction (familywise error, $p<0.05$ ).

We also examined a possible interaction involving all four conditions, using the same cluster-based permutation test, namely on the difference between differences: (pictures-linked - pictures-unlinked) minus (patternslinked - patterns-unlinked).

\section{Source reconstruction}

Following the sensor-level ERF cluster-based statistical analyses, where we controlled for multiple comparisons over sensors and time-points, we then performed a post hoc source reconstruction analysis within the time window already identified at the sensor-level as significant. Source reconstruction, therefore, serves to interrogate the sensor level results further and illustrate the sources of the effect already identified. Consequently, the peaks at the source level are reported without requiring further correction for multiple comparisons over the whole brain (see Gross et al., 2013), as this was already performed at the sensor level. Source reconstruction was performed using the DAiSS toolbox (https://github.com/SPM/DAiSS) included in SPM12. The linearly constrained minimum variance (LCMV) beamformer algorithm (Van Veen et al., 1997) was used to generate maps of power differences between conditions of interest, as informed by the preceding ERF results. For each participant, the covariance matrix was estimated using a common spatial filter for all conditions. For consistency, this was performed within the same broadband frequency spectrum as the ERF analysis $(1-30 \mathrm{~Hz})$. Because of the narrow time window of interest ( $<700 \mathrm{~ms})$, this spatial filter was computed across the entire epoch $(-700-700 \mathrm{~ms})$ including both preresponse and postresponse windows. Whole-brain power images per condition and per participant were subsequently generated within only the shorter interval of interest identified by the ERF analysis. Coregistration to MNI space was performed using a $5-\mathrm{mm}$ volumetric grid and was based on nasion, left and right preauricular fiducials. The forward model was computed using a single-shell head model (Nolte, 2003). This resulted in one weight-normalized image per participant within the interval of interest for each condition, that were then smoothed using a 12-mm Gaussian kernel, and a t-contrast was performed at the second level.

\section{Results}

\section{Behavioral results}

Participants correctly identified images as either being congruent or incongruent with the antecedent clip on an average of $93 \%$ of probe trials (SD 0.75 ), confirming they maintained their attention throughout the scanning session.

\section{Eye tracking results}

Oculomotor behavior was examined during the time window where ERF analyses showed the only significant

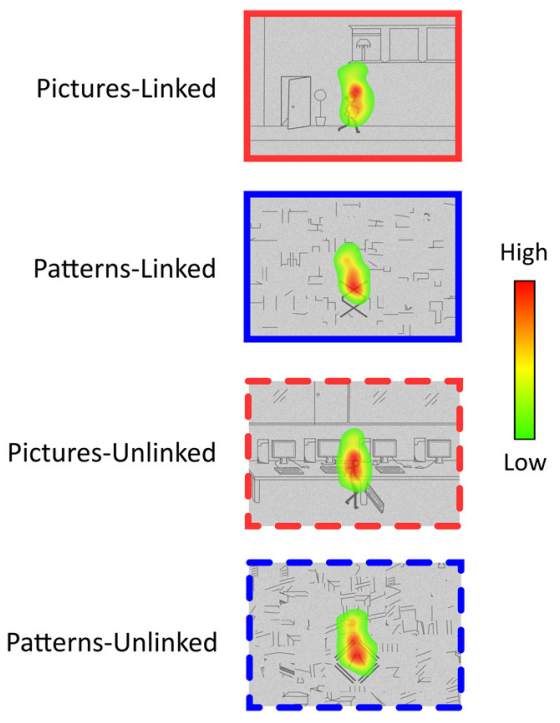

Figure 2. Eye tracking results. Group eye fixation heat maps for each condition during the image 1 time window. Red indicates higher fixation density, and green lower fixation density.

difference between the pictures-linked and patternslinked conditions, which was during the first image. Fixation heat maps revealed the spatial pattern of fixations during image 1 (0-700 ms; Fig. 2) were highly similar across the conditions, and confirmed that participants maintained their focus on the center of the images. No significant difference in fixation count was found between conditions during image $1\left(F_{(3,45)}=0.535, p=0.661\right)$, and this included between pictures-linked and patterns-linked $\left(t_{(15)}=0.141, p=0.889\right)$.

\section{Postscan surprise memory test}

After scanning, participants engaged in a surprise free recall memory test for pictures-linked and patterns-linked movies. A paired samples $t$ test revealed no significant difference in recall (pictures-linked mean 9.48, SD 1.21; patterns-linked mean 9.24, SD $0.89 ; t_{(20)}=0.7555$, $p=0.459$ ). Participants, therefore, successfully encoded both types of event stimuli, although they were never instructed to do so.

\section{ERFs}

The primary focus was on comparing the pictureslinked and patterns-linked conditions. We examined this contrast across all time windows of interest, from the cue preceding the movie, to the final image frame, and similarly for the equivalent gap frames. The only significant difference between pictures-linked and patterns-linked conditions was evident at the very first image, involving one negative cluster emerging between $178-447 \mathrm{~ms}$ $(p=0.0398)$ and distributed across right frontotemporal sensors (Fig. 3A). This difference could not be because of an effect of image type (i.e., pictures) per se, as no difference was observed at image 1 between pictures-unlinked and patterns-unlinked $(p=0.173)$. In fact, it is clear from Figure $3 A$, that pictures-linked sits apart from the other 
A
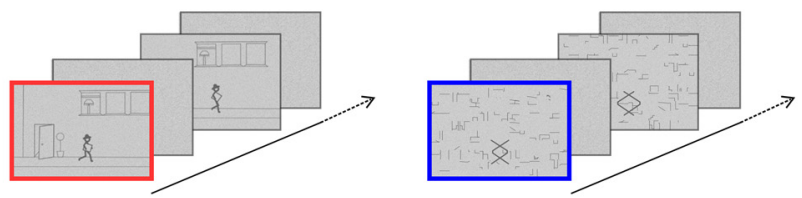

Pictures-Linked vs Patterns-Linked

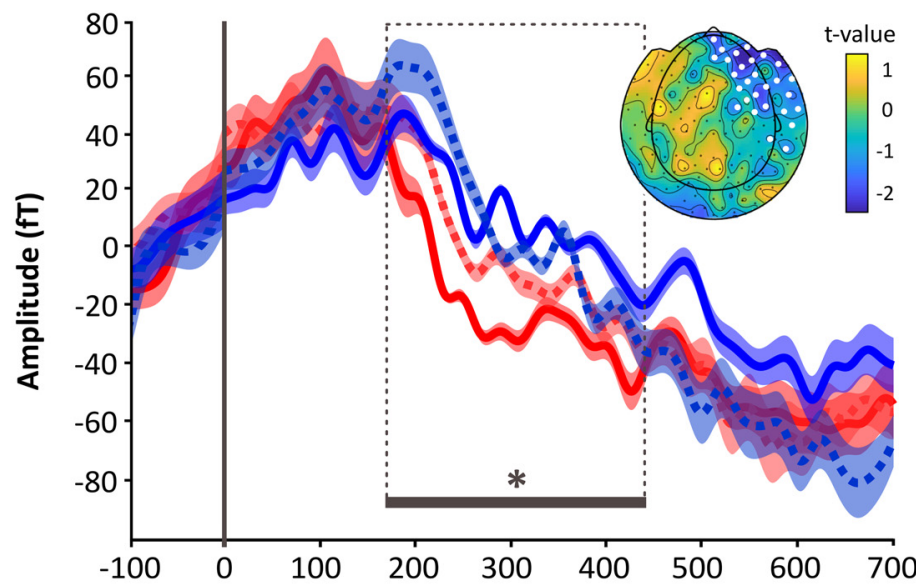

Time from onset of image $1(\mathrm{~ms})$

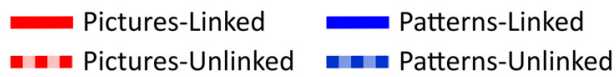

B
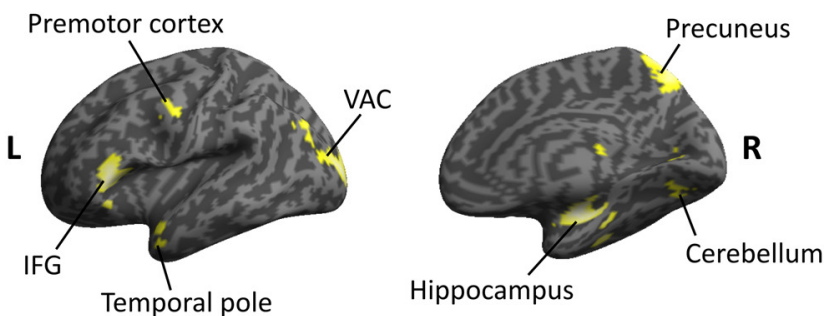

Figure 3. MEG results. A, The ERF analysis revealed a significant difference between pictures-linked (bold red line) and patternslinked (bold blue line) for first image frame between 178 and $447 \mathrm{~ms}\left({ }^{*} p=0.0398\right)$, indicated by the dashed line box. Displayed are the grand-averaged ERFs (shading indicates the SEM) for all four conditions, averaged over a right frontotemporal cluster (marked by white dots on the adjacent topoplot) within which the significant difference between pictures-linked and patterns-linked was observed. Displayed to the right of the ERF panel is the topographic distribution of the difference ( $t$ values), displayed over a helmet layout. Pictures-unlinked is represented by the dashed red line, and patterns-unlinked by the dashed blue line. $\boldsymbol{B}$, Source reconstruction of evoked activity at image 1 during the 178 to $447 \mathrm{~ms}$ interval, displayed on a rendered inflated cortical surface, thresholded at $p<0.005$ uncorrected. L, left hemisphere; R, right hemisphere; IFG, inferior frontal gyrus; VAC, visual association cortex.

three conditions, differing significantly from not only patterns-linked (as already noted), but also patterns-unlinked $(p=0.013)$, and approaching significance for pictures-unlinked $(p=0.0678)$. The other three conditions do not differ from one another: this includes patterns-linked and patterns-unlinked $(p=0.3583)$, and patterns-linked and pictures-unlinked $(p=0.357)$, showing that the effect found cannot be because of linking per se

This pattern of results, with pictures-linked driving the effect at image 1, suggests that there may be an interaction effect across the four conditions during this image frame. When this was tested formally, we found there was indeed a significant interaction effect at image 1 $(p=0.012$; time window $=164-475 \mathrm{~ms})$ encompassing the same time window within which pictures-linked and patterns-linked diverged (see Fig. 3A).

\section{Source reconstruction}

We subsequently performed a beamformer analysis on the image 1 pictures-linked versus patterns-linked contrast, restricted to the same time window (178-447 ms) and frequency band $(1-30 \mathrm{~Hz})$ within which the significant difference in evoked responses was found. This analysis 
served to give a better indication of where in the brain this difference originated. The primary peak difference was found in the right hippocampus for pictures-linked relative to patterns-linked (peak $x, y, z=32,-20$, -16; Fig. 3B), along with the right precuneus $(12,-66,56)$, left visual association cortex $(-22,-96,10)$, left inferior frontal gyrus $(-48,26,2)$, left premotor cortex $(-48,-2,38)$, and right cerebellum $(-30,-64,-58)$.

\section{Discussion}

Unfolding events are central to how we experience the world. In this study we had participants watch dynamic, movie-like events, and compared those built from successively linked scenes (pictures-linked) to those composed of successively linked non-scene patterns (patterns-linked). By using an ERF sliding time window approach to the analysis, we strategically examined image frames across the movies. This novel design allowed a millisecond-by-millisecond examination of the transition from a single image frame to an unfolding event, with a particular interest in hippocampal responses. Only one difference between the closely matched scene and non-scene events emerged, and that was within $178-447 \mathrm{~ms}$ of the onset of the first image frame, detectable across frontotemporal sensors. Further probing of this difference using source reconstruction revealed greater engagement of a set of brain regions across parietal, frontal, premotor, and cerebellar cortices, with the largest change in broadband $(1-30 \mathrm{~Hz})$ power in the hippocampus during pictures-linked events.

A notable feature of the results is that the only difference between scene and non-scene-based events was during viewing of the first image frame, a point at which an event was yet to unfold. Participants were cued before each trial to inform them which condition was to come, but there was no difference apparent between the two conditions during the cue phase. Rather, the two event types diverged only when an event was initiated. This shows that that the ERF difference found at the first movie image did not merely bleed in from the preceding cue period.

A small number of previous MEG studies have investigated the neural correlates of event processing particularly in the form of autobiographical memory recall (Fuentemilla et al., 2014, 2018; Hebscher et al., 2019, 2020; McCormick et al., 2020). Just one of these studies examined the earliest point of event recall initiation (McCormick et al., 2020) and found that within the first $200 \mathrm{~ms}$ of autobiographical event retrieval, the hippocampus was engaged. Another recent MEG study is also relevant. Monk et al. (2021) contrasted the step-by-step building of scene imagery from three successive auditorily-presented object descriptions and an imagined 3D space. This was contrasted with constructing mental images of non-scene arrays that were composed of three objects and an imagined 2D space. They observed a power change in the hippocampus during the initial stage of building scene compared with non-scene imagery. Our finding of an early hippocampal response for the scenebased events aligns with these extant studies.

Why would the difference in hippocampal engagement between scene and non-scene-based events be apparent at the first image frame? One might have expected that it would require events to unfold at least over a couple of image frames for significant changes in neural activity to be evident. However, it may be that the stage was set, so to speak, as soon as the first scene image was viewed. The context of a scene-based event is clearly apparent at that point, and thereafter each additional increment in information provided by subsequent image frames was relatively small. However, the same was true for the nonscene events, and yet the hippocampus was differentially responsive to initial frames of scene-based events. Notably, after the first image frame, the hippocampal response to the subsequent unfolding events was indistinguishable between the two conditions.

Despite the first image frames of pictures-linked and patterns-linked stimuli being composed of the same elements, and both setting a context for an event, it was when the first image resembled the real world that the hippocampal response was elicited. The influence of scene imagery in events is unsurprising given how it mirrors the way in which we experience our surroundings as scene snapshots between blinks and saccades (Clark et al., 2020). Indeed, it has been suggested that one function of the hippocampus may be to support the construction of internal models of the world in the form of scene imagery even during perception (Maguire and Mullally, 2013), and our results are supportive of the link between the hippocampus and scene processing. But what is it about a scene image that provoked the hippocampal response? As noted above, even a single scene can provide a clear indication of the context, and hippocampal engagement may reflect this context being registered.

Further insight may be gained by looking at the pictures-unlinked control condition. In both the pictureslinked condition and the pictures-unlinked control condition a single image could provide a clear indication of a context. Nevertheless, there was a (near-significant) ERF difference between these two conditions at the point of the first image. This suggests that it may be more than the registration of the real-world context that is the influential factor, as contexts were present in both conditions. In the pictures-linked condition, a participant knew that the context depicted in the first image was going to endure for the entire clip, because the cue preceding each clip advised of the upcoming condition. Similarly, they knew that each image in the pictures-unlinked condition related to that image alone, and would not endure across the clip. Consequently, it may be that for the first image in a pictures-linked movie, the context is registered, perhaps a relevant scene template or schema is activated fully (Gilboa and Marlatte, 2017), and then used to help link each image across the sequence. In contrast, the first image in a pictures-unlinked clip may be limited to just registering the context.

Our finding of a very early hippocampal response to unfolding scene-based events differs from fMRI studies of 
movie viewing that found the hippocampus responded later, toward the offset of events, with the speculation that this reflected event replay, aiding memory consolidation (Ben-Yakov and Dudai, 2011; Baldassano et al., 2017; for review, see Griffiths and Fuentemilla, 2020). There are several differences between our study and this previous work. For instance, the latter typically involved explicit memory encoding, participants knew they would be tested afterward, and this may have influenced hippocampal engagement toward the end of events if memory rehearsal occurred. By contrast, our task had no memory demands, although excellent incidental encoding took place. In addition, our study was not designed to assess event boundaries; indeed, our two conditions were very highly matched in terms of event structure, which may have precluded boundary-related findings. Prior studies also used fMRI, which is blind to rapid, phasic neuronal activity, given the slow nature of the hemodynamic response. The few EEG studies that have examined memory encoding using movies were conducted at the sensor level (Silva et al., 2019), and did not source localize responses to specific brain structures. Further MEG studies in the future would be particularly helpful in extending event, and event boundary, research to characterize more precisely the temporal dynamics of hippocampal activity.

Beyond the hippocampus, our results also revealed the involvement of a broader set of brain regions associated with pictures-linked more so than patterns-linked movies, namely, the posterior parietal, inferior frontal, premotor, and cerebellar cortices. Consideration of these areas may shed further light on differences between the two conditions. These brain areas have been identified in numerous studies as part of a network that processes biological motion and the anticipation of incoming intentional movement (Battelli et al., 2003; Rizzolatti and Craighero, 2004; Saygin et al., 2004; Fraiman et al., 2014). In particular, this has been observed in the context of point-light displays, in which a small number of moving lights (e.g., at the joints of a moving person) are sufficient to interpret this as behavior (e.g., dancing). The pictures-linked events were highly simplified portrayals of activities depicted by stick-figures, lines and circles to create simple scenes. Although 2D drawings, they evoked 3D unfolding events of real-world activities that were easily grasped by participants. Scene-based and patternbased evolving stimuli may have been processed differently because abstract patterns were not perceived as intentional, biological stimuli, while participants could automatically infer the actions performed in scenebased events, even as early as the first image frame. Indeed, through piloting we sought to exclude patterns that consistently evoked the sense of biological motion. The success of our efforts was reflected in the descriptions provided by participants in the postscan memory test. For example, elements of a patterns-linked movie showing three overlapping diamond shapes was described as "diamond shapes gradually expanded outwards, then rotated clockwise," while pictures-linked movies were typically described in terms of the intentionality of the stick-figure.
Biological motion is often related to theory of mind. Could theory of mind explain the ERF differences between the pictures-linked and patterns-linked conditions? We feel this is unlikely given that brain areas typically engaged by theory of mind did not emerge in the analyses. Moreover, while biological motion perception appears to relate to some aspects of theory of mind, they are not equivalent constructs (Rice et al., 2016; Meinhardt-Injac et al., 2018). For example, people with theory of mind deficits (e.g., in the context of autism) may demonstrate deficits in the perception of biological motion relative to controls but this may depend on whether emotional state information is required (Todorova et al., 2019). Whether there is a common neural circuitry underlying biological motion and theory of mind remains unclear. It is likely that the ability to perceive biological motion is required to make social judgements, but it is not the sole component of theory of mind processing (Fitzpatrick et al., 2018). We suggest that our simple, emotionally neutral event movies did not necessarily induce theory of mind processes and, consequently, engagement of brain areas associated with theory of mind was not increased for pictures-linked stimuli.

What other alternative explanations might there be for the hippocampal difference between pictures-linked and patterns-linked movies? It could be argued that the effect of pictures-linked was simply the result of scene processing per se. If this was the case, then a difference ought to have been observed between the pictures-unlinked and patterns-unlinked conditions, since the hippocampus is known to respond strongly to scenes relative to nonscene stimuli (Hassabis et al., 2007a; Graham et al., 2010; Zeidman et al., 2015; Hodgetts et al., 2016; Monk et al., 2021); however, no difference was apparent. This suggests that the type of image alone cannot explain the observed hippocampal effect. Another possibility is that linking or sequencing accounts for the finding. However, linking and unfolding sequences were features of both pictures-linked and patterns-linked, and so this factor cannot easily explain the change in hippocampal power. In addition, no significant differences between any other pairs of conditions, including between patterns-linked and patterns-unlinked, and patterns-linked and picturesunlinked were evident, suggesting the effect was not solely explained by the linking of images. It seems that the hippocampus responded to the first scene image only when the expectation was that this picture was the start of a linked, unfolding event, as reflected in the image type by linking interaction that we observed.

Despite the measures taken to closely match event stimuli in terms of their sense of unfolding, scenes could simply be more engaging or predictable than patternbased events. If so, then one might have expected event memory to differ in the surprise postscan test, but it did not, and both types of movie clips were easily recollected as clear narratives. We might also have expected to observe differences in oculomotor behavior, but none were evident, also an indication of similar attentional processes for the two conditions. Consequently, we can conclude that the neural difference identified between the two 
conditions was not because of a large divergence in encoding success. However, we acknowledge that memory differences might emerge with more complex stimuli. Furthermore, events were very well matched in terms of the number of subevents, and their evolving nature as reflected in the highly similar ratings for "linking" and "thinking ahead" measures during piloting. It also seems unlikely that the difference between the two event types can be explained by working memory load. If pictureslinked movies were easier to hold in mind, while patternslinked were more effortful to process, we would have expected this to be reflected at later points in the movie clips, as memory load increased, but no such effect was apparent.

In summary, this MEG study revealed very early hippocampal engagement associated with the viewing of events built from scenes, over and above highly matched evolving sequences built from non-scene imagery. Together with the hippocampus, the involvement of other brain regions, including posterior parietal, inferior frontal, premotor, and cerebellar cortices, may reflect the processing of biologically-relevant information, which typifies the scene-rich episodes we encounter in our daily lives.

\section{References}

Baldassano C, Chen J, Zadbood A, Pillow JW, Hasson U, Norman KA (2017) Discovering event structure in continuous narrative perception and memory. Neuron 95:709-721.e5.

Barry DN, Barnes GR, Clark IA, Maguire EA (2019) The neural dynamics of novel scene imagery. J Neurosci 39:4375-4386.

Battelli L, Cavanagh P, Thornton IM (2003) Perception of biological motion in parietal patients. Neuropsychologia 41:1808-1816.

Ben-Yakov A, Dudai Y (2011) Constructing realistic engrams: poststimulus activity of hippocampus and dorsal striatum predicts subsequent episodic memory. J Neurosci 31:9032-9042.

Betti V, Corbetta M, de Pasquale F, Wens V, Della Penna S (2018) Topology of functional connectivity and hub dynamics in the beta band as temporal prior for natural vision in the human brain. $\mathrm{J}$ Neurosci 38:3858-3871.

Bush D, Bisby JA, Bird CM, Gollwitzer S, Rodionov R, Diehl B, McEvoy AW, Walker MC, Burgess N (2017) Human hippocampal theta power indicates movement onset and distance travelled. Proc Natl Acad Sci USA 114:12297-12302.

Cabeza R, St Jacques P (2007) Functional neuroimaging of autobiographical memory. Trends Cogn Sci 11:219-227.

Chang WT, Jääskeläinen IP, Belliveau JW, Huang S, Hung AY, Rossi S, Ahveninen J (2015) Combined MEG and EEG show reliable patterns of electromagnetic brain activity during natural viewing. Neuroimage 114:49-56.

Chen Y, Farivar R (2020) Natural scene representations in the gamma band are prototypical across subjects. Neuroimage 221:117010.

Clark IA, Monk AM, Maguire EA (2020) Characterizing strategy use during the performance of hippocampal-dependent tasks. Front Psychol 11:2119.

Colgin LL (2013) Mechanisms and functions of theta rhythms. Annu Rev Neurosci 36:295-312.

Colgin LL (2016) Rhythms of the hippocampal network. Nat Rev Neurosci 17:239-249.

Crespo-García M, Zeiller M, Leupold C, Kreiselmeyer G, Rampp S, Hamer HM, Dalal SS (2016) Slow-theta power decreases during item-place encoding predict spatial accuracy of subsequent context recall. Neuroimage 142:533-543.

Cutting JE (2005) Perceiving scenes in film and in the world. In: Moving image theory: ecological considerations (Anderson JD,
Anderson BF, eds), pp 9-27. Carbondale: University of Southern Illinois Press.

Dede AJO, Frascino JC, Wixted JT, Squire LR (2016) Learning and remembering real-world events after medial temporal lobe damage. Proc Natl Acad Sci USA 113:13480-13485.

Doeller CF, King JA, Burgess N (2008) Parallel striatal and hippocampal systems for landmarks and boundaries in spatial memory. Proc Natl Acad Sci USA 105:5915-5920.

Fitzpatrick P, Frazier JA, Cochran D, Mitchell T, Coleman C, Schmidt RC (2018) Relationship between theory of mind, emotion recognition, and social synchrony in adolescents with and without autism. Front Psychol 9:1337.

Fraiman D, Saunier G, Martins EF, Vargas CD (2014) Biological motion coding in the brain: analysis of visually driven EEG functional networks. PLoS One 9:e84612.

Fuentemilla L, Barnes GR, Düzel E, Levine B (2014) Theta oscillations orchestrate medial temporal lobe and neocortex in remembering autobiographical memories. Neuroimage 85:730-737.

Fuentemilla L, Palombo DJ, Levine B (2018) Gamma phase-synchrony in autobiographical memory: evidence from magnetoencephalography and severely deficient autobiographical memory. Neuropsychologia 110:7-13.

Gilboa A, Marlatte H (2017) Neurobiology of schemas and schemamediated memory. Trends Cogn Sci 21:618-631.

Graham KS, Barense MD, Lee ACH (2010) Going beyond LTM in the MTL: a synthesis of neuropsychological and neuroimaging findings on the role of the medial temporal lobe in memory and perception. Neuropsychologia 48:831-853.

Griffiths BJ, Fuentemilla L (2020) Event conjunction: how the hippocampus integrates episodic memories across event boundaries. Hippocampus 30:162-171.

Gross J, Baillet S, Barnes GR, Henson RN, Hillebrand A, Jensen O, Jerbi K, Litvak V, Maess B, Oostenveld R, Parkkonen L, Taylor JR, van Wassenhove V, Wibral M, Schoffelen J-M (2013) Good practice for conducting and reporting MEG research. Neuroimage 65:349-363.

HansImayr S, Staudigl T (2014) How brain oscillations form memories - a processing based perspective on oscillatory subsequent memory effects. Neuroimage 85:648-655.

Hassabis D, Kumaran D, Maguire EA (2007a) Using imagination to understand the neural basis of episodic memory. J Neurosci 27:14365-14374.

Hassabis D, Kumaran D, Vann SD, Maguire EA (2007b) Patients with hippocampal amnesia cannot imagine new experiences. Proc Natl Acad Sci USA 104:1726-1731.

Hasson U, Furman O, Clark D, Dudai Y, Davachi L (2008) Enhanced intersubject correlations during movie viewing correlate with successful episodic encoding. Neuron 57:452-462.

Hebscher M, Meltzer JA, Gilboa A (2019) A causal role for the precuneus in network-wide theta and gamma oscillatory activity during complex memory retrieval. Elife 8:e43114.

Hebscher M, Ibrahim C, Gilboa A (2020) Precuneus stimulation alters the neural dynamics of autobiographical memory retrieval. Neuroimage 210:116575.

Hillebrand A, Barnes GR (2002) A quantitative assessment of the sensitivity of whole-head MEG to activity in the adult human cortex. Neuroimage 16:638-650.

Hodgetts CJ, Shine JP, Lawrence AD, Downing PE, Graham KS (2016) Evidencing a place for the hippocampus within the core scene processing network. Hum Brain Mapp 37:3779-3794.

Kaplan R, Doeller CF, Barnes GR, Litvak V, Düzel E, Bandettini PA, Burgess N (2012) Movement-related theta rhythm in humans: coordinating self-directed hippocampal learning. PLoS Biol 10: e1001267.

Kumaran D, Maguire EA (2006) The dynamics of hippocampal activation during encoding of overlapping sequences. Neuron 49:617629.

Kurczek J, Wechsler E, Ahuja S, Jensen U, Cohen NJ, Tranel D, Duff M (2015) Differential contributions of hippocampus and medial 
prefrontal cortex to self-projection and self-referential processing. Neuropsychologia 73:116-126.

Lankinen K, Saari J, Hari R, Koskinen M (2014) Intersubject consistency of cortical MEG signals during movie viewing. Neuroimage 92:217-224.

Lehn H, Steffenach HA, van Strien NM, Veltman DJ, Witter MP, Håberg AK (2009) A specific role of the human hippocampus in recall of temporal sequences. J Neurosci 29:3475-3484.

Liu Y, Dolan RJ, Kurth-Nelson Z, Behrens TEJ (2019) Human replay spontaneously reorganizes experience. Cell 178:640-652.

Magliano JP, Zacks JM (2011) The impact of continuity editing in narrative film on event segmentation. Med J Aust 194:180-185.

Maguire EA (2001) Neuroimaging studies of autobiographical event memory. Philos Trans R Soc Lond B Biol Sci 356:1441-1451.

Maguire EA, Mullally SL (2013) The hippocampus: a manifesto for change. J Exp Psychol Gen 142:1180-1189.

Maris E (2004) Randomization tests for ERP topographies and whole spatiotemporal data matrices. Psychophysiology 41:142-151.

Maris E (2012) Statistical testing in electrophysiological studies. Psychophysiology 49:549-565.

Maris E, Oostenveld R (2007) Nonparametric statistical testing of EEG- and MEG-data. J Neurosci Methods 164:177-190.

Mayes AR, Isaac CL, Holdstock JS, Hunkin NM, Montaldi D, Downes JJ, Macdonald C, Cezayirli E, Roberts JN (2001) Memory for single items, word pairs, and temporal order of different kinds in a patient with selective hippocampal lesions. Cogn Neuropsychol 18:97123.

McCormick C, Barry DN, Jafarian A, Barnes GR, Maguire EA (2020) vmPFC drives hippocampal processing during autobiographical memory recall regardless of remoteness. Cereb Cortex 30:59725987.

Meinhardt-Injac B, Daum MM, Meinhardt G, Persike M (2018) The two-systems account go theory of mind: testing the links to socialperceptual and cognitive abilities. Front Hum Neurosci 12:25.

Meyer SS, Rossiter H, Brookes MJ, Woolrich MW, Bestmann S, Barnes GR (2017) Using generative models to make probabilistic statements about hippocampal engagement in MEG. Neuroimage 149:468-482.

Mikuni N, Nagamine T, Ikeda A, Terada K, Taki W, Kimura J, Kikuchi H, Shibasaki H (1997) Simultaneous recording of epileptiform discharges by meg and subdural electrodes in temporal lobe epilepsy. Neuroimage 5:298-306.

Monk AM, Dalton MA, Barnes GR, Maguire EA (2021) The role of hippocampal-ventromedial prefrontal cortex neural dynamics in building mental representations. J Cogn Neurosci 33:89-103.

Nolte G (2003) The magnetic lead field theorem in the quasi-static approximation and its use for magnetoencephalography forward calculation in realistic volume conductors. Phys Med Biol 48:3637-3652.

Oostenveld R, Fries P, Maris E, Schoffelen JM (2011) FieldTrip: open source software for advanced analysis of MEG, EEG, and invasive electrophysiological data. Comput Intell Neurosci 2011:156869.

Pu Y, Cheyne DO, Cornwell BR, Johnson BW (2018) Non-invasive investigation of human hippocampal rhythms using magnetoencephalography: a review. Front Neurosci 12:273.

Reagh ZM, Delarazan Al, Garber A, Ranganath C (2020) Aging alters neural activity at event boundaries and posterior medial network. Nat Comm 11:3980.
Rice K, Anderson LC, Velnoskey K, Thompson JC, Redcay E (2016) Biological motion perception links diverse facets of theory of mind during middle childhood. J Exp Child Psychol 146:238-246.

Rizzolatti G, Craighero L (2004) The mirror-neuron system. Annu Rev Neurosci 27:169-192.

Rosenbaum RS, Köhler S, Schacter DL, Moscovitch M, Westmacott R, Black SE, Gao F, Tulving E (2005) The case of K.C.: contributions of a memory-impaired person to memory theory. Neuropsychologia 43:989-1021.

Ruzich E, Crespo-García M, Dalal SS, Schneiderman JF (2019) Characterizing hippocampal dynamics with meg: a systematic review and evidence-based guidelines. Hum Brain Mapp 40:13531375.

Saygin AP, Wilson SM, Hagler DJ, Bates E, Sereno MI (2004) Pointlight biological motion perception activates human premotor cortex. J Neurosci 24:6181-6188.

Scoville WB, Milner B (1957) Loss of recent memory after bilateral hippocampal lesions. J Neurol Neurosurg Psychiatry 20:11-21.

Schapiro AC, Turk-Browne NB, Norman KA, Botvinick MM (2016) Statistical learning of temporal community structure in the hippocampus. Hippocampus 26:3-8.

Shigeto H, Morioka T, Hisada K, Nishio S, Ishibashi H, Kira D, Tobimatsu S, Kato M (2002) Feasibility and limitations of magnetoencephalographic detection of epileptic discharges: simultaneous recording of magnetic fields and electrocorticography. Neurol Res 24:531-536.

Silva M, Baldassano C, Fuentemilla L (2019) Rapid memory reactivation at movie event boundaries promotes episodic encoding. $J$ Neurosci 39:8538-8548.

Spreng RN, Mar RA, Kim ASN (2009) The common neural basis of autobiographical memory, prospection, navigation, theory of mind, and the default mode: a quantitative meta-analysis. J Cogn Neurosci 21:489-510.

Summerfield JJ, Hassabis D, Maguire EA (2010) Differential engagement of brain regions within a "core" network during scene construction. Neuropsychologia 48:1501-1509.

Svoboda E, McKinnon MC, Levine B (2006) The functional neuroanatomy of autobiographical memory: a meta-analysis. Neuropsychologia 44:2189-2208

Tan ES (2018) A psychology of the film. Palgrave Commun 4:82.

Todorova GK, Hatton REM, Pollick FE (2019) Biological motion perception in autism spectrum disorder: a meta-analysis. Mol Autism 10:49.

Van Veen BD, Van Drongelen W, Yuchtman M, Suzuki A (1997) Localization of brain electrical activity via linearly constrained minimum variance spatial filtering. IEEE Trans Biomed Eng 44:867880.

Wager TD, Keller MC, Lacey SC, Jonides J (2005) Increased sensitivity in neuroimaging analyses using robust regression. Neuroimage 26:99-113.

Zacks JM, Tversky B, lyer G (2001) Perceiving, remembering, and communicating structure in events. J Exp Psychol Gen 130:29-58.

Zeidman P, Mullally SL, Maguire EA (2015) Constructing, perceiving, and maintaining scenes: hippocampal activity and connectivity. Cereb Cortex 25:3836-3855. 